\title{
Melanoma: tumor microenvironment and new treatments*
}

\author{
Mara Huffenbaecher Giavina-Bianchi ${ }^{1}$ \\ Cyro Festa Neto ${ }^{3}$
}

Pedro Francisco Giavina-Bianchi Junior ${ }^{2}$

DOI: http:/ /dx.doi.org/10.1590/abd1806-4841.20176183

\begin{abstract}
In the recent past years, many discoveries in the tumor microenvironment have led to changes in the management of melanoma and it is rising up hopes, specially, to those in advanced stages. FDA approved seven new drugs from 2011 to 2014. They are: Vemurafenib, Dabrafenib and Trametinib, kinases inhibitors used for patients that have BRAFV600E mutation; Ipilimumab (anti-CTLA4), Pembrolizumab (anti-PD-1) and Nivolumab (anti-PD-1), monoclonal antibodies that stimulate the immune system; and Peginterferon alfa-2b, an anti-proliferative cytokine used as adjuvant therapy. In this article, we will review the molecular bases for these new metastatic melanoma therapeutic agents cited above and also analyze new molecular discoveries in melanoma study, as Cancer-Testis antigens (CT). They are capable of induce humoral and cellular immune responses in cancer patients and because of this immunogenicity and their restrict expression in normal tissues, they are considered an ideal candidate for vaccine development against cancer. Among CT antigens, NY-ESO-1 is the best characterized in terms of expression patterns and immunogenicity. It is expressed in $20-40 \%$ of all melanomas, more in metastatic lesions than in primary ones, and it is very heterogeneous inter and intratumoral. Breslow index is associate with NY-ESO-1 expression in primary cutaneous melanomas, but its relation to patient survival remains controversial.
\end{abstract}

Keywords: Immunotherapy; Melanoma; Molecular targeted therapy; Tumor microenvironment

\section{INTRODUCTION}

Melanoma has received considerable attention and has been addressed in several studies due to the increase in incidence and mortality rates worldwide, especially in individuals with white skin. ${ }^{1,2}$ In Brazil, there will be 3 to 4 new cases/ 100,000 inhabitants according to the National Cancer Institute (INCA) in 2016, totaling 5,670 new cases. ${ }^{3}$ In the US, 76,380 new cases of melanoma are estimated this year. ${ }^{4}$

The various findings of recent years through studies of the tumor microenvironment already cause changes in the therapeutic approach and raise hopes for better management of patients with melanoma, especially those in the more advanced stages of the disease. The approval of seven new drugs for metastatic melanoma by the FDA between 2011 and 2014 is impressive. The entry of these drugs into the market is certainly the result of numerous projects both in the basic area of research, with the discovery of molecular aspects of melanoma, and in clinical trials in which the safety and efficacy of these drugs have been proven. These are: Vemurafenib, Dabrafenib and Trametinib, which are inhibitors of kinases used in patients with a BRAFV600E mutation; Ipilimumab (anti-CTLA4), Pembrolizumab (anti-PD-1) and Nivolumab (anti-PD-1), which are monoclonal antibodies that stimulate the immune system; and
Peginterferon alfa-2b, which is an antiproliferative cytokine used as adjuvant therapy.

Tumors sometimes express, at a certain stage of their development, anomalous proteins that may be targets for diagnostic methods and therapeutic interventions. Immunotherapy against cancer is considered a promise of effective and specific treatment for this disease and has been addressed to date with passive antibody transfer, adoptive transfer of $\mathrm{T}$ cells and therapeutic vacines. ${ }^{5}$

In this article, we review the molecular basis of these new treatments for metastatic melanoma and also other molecular discoveries, such as the so-called Cancer-Testis (CT) antigens, still in clinical trials for their use in vaccines.

\section{ANTITUMORAL RESPONSE AND TUMOR-INFILTRATING LYMPHOCYTES (TIL)}

In the event of the onset and development of tumors, the immune system is exposed to numerous previously unseen antigens that are derived from genetic abnormalities. In this context, the immune system can recognize and eliminate some tumors at an early stage of their development. The adaptive immune system ap-

Received on 20.06.2016.

Approved by the Advisory Board and accepted for publication on 28.08.2016.

* Study conducted at Dermatology Department of University of São Paulo Medical School (FMUSP) - São Paulo (SP), Brazil.

Financial support: none.

Conflict of interest: none

Dermatology Department of the Hospital das Clínicas of the Universidade de São Paulo Medical School (FMUSP) - São Paulo (SP), Brazil.

Disciplines of Allergy and Immunology of the Universidade de São Paulo Medical School (FMUSP) - São Paulo (SP), Brazil.

Dermatology Department of Universidade de São Paulo Medical School (FMUSP) - São Paulo (SP), Brazil.

(O2017 by Anais Brasileiros de Dermatologia 
pears to be of fundamental importance in the antitumor response, which is triggered by activation of a wide range of diverse and highly specific receptors on $\mathrm{T}$ and $\mathrm{B}$ cells.

An effective immune response begins when the T or B cell recognizes the tumor antigen in a pro-stimulatory context and undergoes activation and proliferation. B cells have as receptor a surface IgM immunoglobulin and are able to recognize soluble antigens, bind to them and differentiate into plasmocytes, which secrete large amounts of highly specific antibodies. Traditional T cells (alpha-beta receptor) recognize antigens in the form of small peptides, presented through MHC molecules on the surface of antigen-presenting cells and tumor cells. The T lymphocyte receptor is associated with the CD3 surface molecule that is a marker, regardless of whether this cell is helper, cytotoxic or regulatory.

$\mathrm{CD} 4+\mathrm{T}$ cells bind to antigens presented by MHC class II molecules, which are expressed primarily by antigen-presenting cells. CD8+ $\mathrm{T}$ cells bind to complexes formed by antigens and MHC class I molecules, which are present in all nucleated cells, including antigen-presenting cells. CD4+ cells, following their binding to antigen-presenting cells, can differentiate into many types of effector cells, depending on the cytokine combination of the microenvironment. One possibility is that it becomes a CD4+ helper $\mathrm{T}$ (Th) cell and, as the name says, it helps activated B cells, NK (natural killer) cells and cytotoxic lymphocytes $(\mathrm{CD} 8+\mathrm{T}) \cdot \mathrm{CD} 8+\mathrm{T}$ cells, after being activated by antigen-presenting cells, are responsible for exerting cytotoxic effects directly on tumor cells and therefore play a central role in the antitumor immune response, controlling the growth and spread of cancer. ${ }^{6-8}$

When the tissue microenvironment is not inflammatory, the $\mathrm{T}$ cell can differentiate into regulator (Treg), which is important in inhibiting the immune system. Treg cells are a very interesting and promising target in immunotherapy for cancer because they are often present in the tumor, inhibiting the adaptive and innate responses of the host. Treg cells characteristically express the FoxP3 (transcription factor forkhead box protein 3) molecule, which belongs to a large family of different transcription factors, whose function would be primarily regulatory of the immune system. However, CD8+ FoxP3+ cells (double positive) have also been described, whose function has been cytotoxic and non-regulatory, as was imagined. ${ }^{9}$

The presence of tumor-infiltrating lymphocytes (TIL) in melanoma has been associated with better prognosis in several studies and is interpreted as an indicator that the host is promoting a more effective immune response against the tumor. ${ }^{10-18}$ However, other studies have failed to prove this association and the meaning of TIL remains controversial. Gooden et al., in 2011, conducted a meta-analysis on the prognostic influence of TIL on cancers. ${ }^{19}$ The presence of CD3+ and CD8+ cells had a beneficial effect on patient survival, whereas FoxP3 + regulatory cells had no association with overall patient survival. When $\mathrm{CD} 8+$ /FoxP3+ ratio (effector/regulator) was higher, the association was even greater. However, more studies are needed to corroborate these findings.

\section{NEW THERAPIES FOR METASTATIC MELANOMA AND THEIR MOLECULAR CHANGES}

The drugs approved by the FDA for metastatic melanoma between 2011-2014 and their mechanism of action are summarized in table 1.

\section{Target-therapy}

Target therapies are drugs or other substances that block the growth or spread of a cancer by interfering with specific molecules (target molecules) that are involved in the growth, progression, and spread of cancer. ${ }^{20}$ They differ from chemotherapy in several ways:

- they act on specific molecules associated with cancer, while most standard chemotherapeutics act on all rapidly dividing cells, whether normal or cancerous.

- they are deliberately chosen or designed to interact with their target, while most chemotherapeutics have been identified because of their ability to kill cells.

- they are often cytostatic (block tumor proliferation) while standard chemotherapy agents are cytotoxic (kill the tumor cell).

Mutations in somatic oncogenes were identified in subgroups of patients with melanoma (Table 2). Among them all, the mutations in the pathway genes and the MAP kinase pathway transduction signals are prominent in the pathogenesis of cutaneous melanoma. When activated, this mutation promotes proliferation, increased survival, and invasive power of tumor cells. The main mutant gene of this pathway is BRAF. ${ }^{21}$ The drugs, when competing with this mutation, have a direct antiproliferative effect, leading to regression or, at least, non-proliferation of cancer.

Vemurafenib is an oral, highly selective and competitive drug with the BRAFV600E mutation, which was approved by the FDA in 2011 and by ANVISA in January 2012. Used at the dose of $960 \mathrm{mg}$ orally twice daily, it is the first-line of treatment for patients with this mutation in metastatic melanoma. ${ }^{22}$ It can present several adverse events, such as arthralgia, rash, fatigue, alopecia, photosensitivity, squamous cell carcinomas and keratoacanthomas.

Dabrafenib is a selective and reversible inhibitor of the BRAFV600E mutation. It was approved by the FDA in 2013 and by ANVISA in January 2016. It is also administered orally at $150 \mathrm{mg}$ dose twice daily. The most common adverse events are: hyperkeratosis, headache, pyrexia, arthralgia and cutaneous papillomas. ${ }^{23}$

Trametinib is a small molecule, selective inhibitor of MEK1 and MEK2 (BRAFV600E and V600K). It was approved by the FDA

TABLE 1: Main drugs approved by the FDA between 2011 and 2014 for the treatment of metastatic melanoma and their mechanisms of action

\begin{tabular}{lll} 
Mechanisms of action & Drug & Site of action \\
\hline Kinase inhibitor & Vemurafenib & BRAFV600E \\
Kinase inhibitor & Dabrafenib & BRAFV600E \\
Kinase inhibitor & Trametinib & $\begin{array}{l}\text { BRAFV600E; } \\
\text { V600K }\end{array}$ \\
Anti-CTLA4 & Ipilimumab & CD80 and CD86 \\
Anti-PD-1 & Pembrolizumab & PD-L1 and PD-L2 \\
Anti-PD-1 & Nivolumab & PD-L1 and PD-L2 \\
Antiproliferative & $\begin{array}{l}\text { Peginterferon } \\
\text { alfa } 2 b\end{array}$ & Tumor cells
\end{tabular}




\section{TABLE 2: Oncogenes related to different types of melanoma to date}

\begin{tabular}{ll} 
Type of melanoma & Common genetic mutations \\
\hline Cutaneous & BRAF 50\% (80\% V600E, 16\% V600K, \\
& N $\%$ V $600 \mathrm{G} / \mathrm{R})$ \\
& c-KIT $10 \%, \mathrm{BRAF} / \mathrm{NRAS}<10 \%$ \\
Acral / Mucous & GNAQ $45 \%$, GNA11 $60 \%<10 \%$
\end{tabular}

in 2013 but it has not yet been approved by ANVISA. It is administered orally, at $2 \mathrm{mg}$ dose, once daily. Rash, fatigue, diarrhea, peripheral edema, blurred vision, chorioretinopathy and decreased cardiac ejection fraction are possible adverse events. ${ }^{24}$

In addition to the adverse events, a major obstacle to the treatment with these drugs is the occurrence of clinical resistance, observed practically on a universal basis. Primary resistance (not being effective in the first cycle), observed in approximately $15 \%$ of patients, is less frequent than secondary resistance, when the disease progresses after having had an initial improvement, which happens in the majority of patients. ${ }^{25}$

\section{IMUNOTHERAPY}

By inhibiting immune system checkpoints, immunotherapeutics increase or stimulate "de novo" the antitumor response to kill cancer cells rather than acting directly on them, as do chemotherapeutics and target therapies.

\section{Anti-CTLA4 (Ipilimumab)}

This was the first T-cell receptor to be targeted in the clinical treatment of metastatic melanoma. It was approved by the FDA in 2011 and by ANVISA in July 2012. Ipilimumab is a monoclonal antibody intravenously administered at a dose of $3 \mathrm{mg} / \mathrm{kg}$ every 3 weeks, in a total of 4 doses over 3 months. The role of CTLA4 is to regulate the amplitude of $\mathrm{T}$ lymphocyte activation in the beginning of the process. Thus, it uses a costimulation receptor, the CD28. ${ }^{26-28}$ Both share the same ligand CD80 and CD86 (also known as B7.1 and B7.2, respectively). ${ }^{29-32}$ Although the exact mechanism is not yet known, the CTLA4 receptor, when activated, blocks the activation of $\mathrm{T}$ lymphocytes. It seems that its major role is in decreasing the activation of helper T lymphocytes and increasing the regulatory action of regulatory $\mathrm{T}$ lymphocytes. Therefore, by inhibiting lymphocyte blockade, the immune system is stimulated to fight the tumor.

More impressive than the benefit in the mean survival of patients was the maintenance of the therapeutic effects for more than 2 years in $18 \%$ of the cases, even with the suspension of the treatment. This would make us suppose that there could be a re-education of the immune system, which would keep the tumor under control. ${ }^{33}$

The most important adverse events are autoimmune, such as colitis, hepatitis, skin rash, pneumonitis, neuritis, endocrinopathies and ocular problems. ${ }^{33-37}$

\section{Anti-PD-1}

The first PD-1 inhibitor approved by the FDA in 2014 was Pembrolizumab $(10 \mathrm{mg} / \mathrm{kg}$ administered intravenously every 2 or 3 weeks). Then, in the same year, Nivolumab $(3 \mathrm{mg} / \mathrm{kg}$ administered intravenously every 2 weeks) was launched. In Brazil, ANVISA approved Nivolumab in April 2016, and Pembrolizumab was recently registered. Adverse events are less intense than those caused by Ipilimumab and, in order of frequency, are: fatigue, diarrhea, rash, muscle pain, pruritus and nausea. PD-1 axis appears to have an impact on the effector (peripheral) and, therefore, later phase of the immune response. PD-1 is activated in T cells in response to chronic infections, limiting the risk of autoimmunity. ${ }^{38,39}$ PD-1 ligands are two: PD-L1 and PD-L2, which can be expressed by tumor cells, as well as other cells of the hematopoietic or non-hematopoietic lineages. Inflammatory cytokines, such as gamma interferon, may induce the expression of PD-L1 and PD-L2..$^{40}$ PD-1, when binding to its ligands, sends an inhibitory signal to the T cell. Over time, this pathway can lead to T-cell anergy and blockage of the antitumor response. The expression of the PD-1 ligands by the tumor ends up being a mechanism of evasion of the immune response within the tumor microenvironment. ${ }^{38}$

The adverse events of both antibodies in this group are very similar to those of Ipilimumab, but pneumonitis appears to be more prevalent than colitis in anti-PD-1 drugs. ${ }^{41}$

\section{Anti-PD-L1}

Although not yet approved by the FDA, this form of immunotherapy has at least 3 compounds under study aiming at blocking PD-1 axis by inhibiting PD-1, which is one of its ligands. Initially, melanomas, in addition to ovarian and lung cancers, were reported to have a high level of PD-L1 expression in their tumor cells. ${ }^{42-44}$ Later, studies with many other cancers also demonstrated its presence. ${ }^{40}$ There appear to be a strong correlation between the expression of PD-L1 on the surface of the tumor cell with the tumor-infiltrating lymphocytes and the expression of gamma interferon intratumor. This correlation was not only seen with tumors but also at a regional level, where regions with more infiltrates were also the ones that most expressed PD-L1 in the tumor. ${ }^{45}$

The other PD-1 ligand, PD-L2, is highly expressed in certain B-cell lymphomas, such as primary mediastinal B-cell lymphoma, follicular B-cell lymphoma, and Hodgkin lymphoma. ${ }^{46}$ An anti-PD-L1 antibody blocks the interaction between PD-L1 and CD80 and between PD-1 and PD-L1, but does not prevent PD-1 from binding to PD-L2 (something that would only occur with anti- PD-1 antibodies). Thus, according to the dominant interaction in a particular cancer, we could choose one or the other antibody, or even both (Figure 1). ${ }^{36}$

\section{ANTIPROLIFERATIC CYTOKINES: PEGINTERFERON ALFA 2 B}

Interferon, as a single agent, showed only minor antitumor activity. By modifying the pharmacokinetics of IFN-alpha $2 b$ by adding to the $12 \mathrm{kDa}$ polyethylene glycol chain, the interferon molecule is protected from proteolytic breakdown and an increase in half-life occurs.

An European clinical trial in patients with stage III melanoma found that the best results occurred in the subgroup with microscopic involvement of the lymph nodes and with ulcerated tumors. 
It was approved by the FDA in 2011 and it is administered subcutaneously at a dose of $6 \mu \mathrm{g} / \mathrm{kg} /$ week for 8 weeks, and then reduced to a dose of $3 \mu \mathrm{g} / \mathrm{kg} /$ week up to a maximum of 252 weeks. There was no improvement in overall survival, but only in relapse free survival (RFS). The most common adverse events are: leukopenia (reversible with suspension), fatigue, increased AST and ALT, pyrexia, headache, myalgia, tremor and injection site reactions. ${ }^{47}$

\section{Cancer-Testis Antigens}

Identification of a significant number of tumor antigens has become possible since 1990 due to the establishment of in vitro autologous systems and the development of methodologies such as "T cell epitope cloning" and SEREX ("Serological identification of recombinant antigens expressed in cDNA Libraries of human tumors"). Such methodologies allow the screening of proteins expressed by tumor cells capable of inducing a humoral and cellular immune response in cancer patients. Tumor antigens known to date can be grouped into 5 major categories: differentiation antigens, antigens corresponding to mutated proteins, overexpressed or amplified antigens, antigens of viral origin, and Cancer-Testis (CT) antigens. ${ }^{48}$

$\mathrm{CT}$ antigens are generally encoded by genes on the $\mathrm{X}$ chromosome and have as their main characteristic their restricted expression in germ cells of the adult testis, fetal ovary and trophoblast, among normal tissues, and aberrant expression in tumors of different histological types. ${ }^{49-51}$ To date, more than 90 CT antigens are known, grouped into approximately 40 gene families. Several monoclonal antibodies for in situ detection of these antigens have been developed in recent years. ${ }^{52}$

$\mathrm{CT}$ antigens are capable of inducing a humoral and cellular immune response in cancer patients and, because of this immunogenicity and the restricted pattern of expression in normal tissues, are considered ideal candidates for the development of therapeutic vaccines against cancer. This is because testis cells do not express HLA class I molecules and thus do not have antigens for cytotoxic T lymphocytes. This characteristic, added to the physical blockade imposed by the hematopoietic barrier, which prevents the passage of antibodies to the testis, makes it an immunoprivileged, i.e., immunologically protected tissue. Thus, at first, vaccines targeting these antigens should not trigger immune response against the expressed protein in the testis, ensuring that only the tumor cells are destroyed..$^{53-55}$

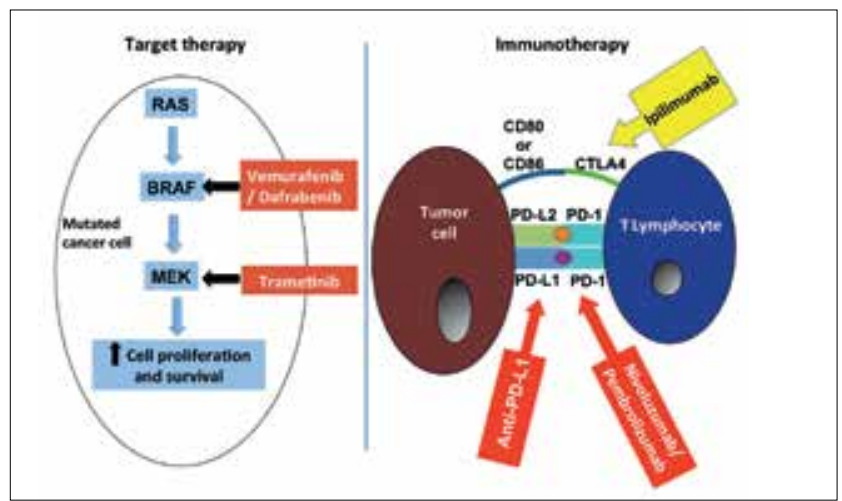

FIGURE 1: Illustration with the main points of action of drugs for the treatment of metastatic melanoma and their sites of action

\section{Antigen NY-ESO-1}

The NY-ESO-1 antigen is one of the most well characterized $\mathrm{CT}$ antigens in terms of expression pattern and immunogenicity. It belongs to the Cancer-Testis CT6 antigen family (it was thus named because it was identified in New York [NY], at the Ludwig Institute for Cancer Research in 1997, in a patient with ESO, i.e., esophagus cancer). ${ }^{52}$ The number 1 stands for the fact that it was the first discovered member of a new family. The antigen was identified by the SEREX technique. In this methodology, a protein expression library produced from cDNA from an esophageal tumor was screened with autologous serum from patients, allowing the identification of antigens recognized by the humoral immune system. ${ }^{48}$ In the following year, Jager et al. were able to isolate CD8 cells from a melanoma patient in who they specifically recognized the NY-ESO-1 antigen. ${ }^{56}$

The main product of NY-ESO-1 gene coding is a $180 \mathrm{ami}-$ no acid protein, with a $\mathrm{N}$-terminal glycine rich and extremely hydrophobic C-terminal region, so insoluble that it could be confused with a transmembrane domain, which there is no evidence. ${ }^{48}$ The antigen has mainly cytoplasmic expression.

Little is known about its function and its involvement in the tumor genesis. This lack of information is due to the fact that there are no corresponding genes in model organisms, such as drosophila and rodents, which makes it impossible to perform knockout tests. $\mathrm{CT}$ antigens are mostly exclusive to primates, making it difficult to conduct pre-clinical trials of therapeutic vaccines in animal models. ${ }^{57}$

In normal human tissues, the expression of NY-ESO-1 is restricted to spermatogonia of the adult testis and gradually decreases with maturation and differentiation of germ cells. It is detected in $20 \%$ to $30 \%$ of lung, esophagus, liver, stomach, prostate, ovary, bladder and melanoma tumors (see below). ${ }^{43,58-63}$ Although rare, but very aggressive, synovial sarcoma presents about $80 \%$ positivity for NY-ESO-1, with an intense and homogeneous distribution throughout the tumor. ${ }^{64}$ In myxoid and round cell liposarcomas, its expression is around $90 \% .{ }^{65}$ However, its frequency is low in colorectal tumors, pancreas tumors, kidney tumors and lymphomas. ${ }^{66-69}$

\section{Antigen NY-ESO-1 in melanoma}

The analysis of the molecular aspects of melanoma has assumed increasing importance for the understanding of its various subtypes, aiming at a more specific treatment for the different phenotypes, and at a better outcome. Regarding the analysis of NYESO-1 in benign melanocytic tumors, two studies were conducted with 19 and 20 nevi each, and in none of them there was expression of this antigen, whether dysplastic, dermal, Spitz, junctional or compound nevi. ${ }^{70,71}$ It was postulated by the authors that NY-ESO-1 could aid in the differential diagnosis between benign nevi and melanomas.

In a recent study, only an in situ melanoma expressed the NY-ESO-1 protein $(1 / 10)$ and the melanomas in the radial growth phase were all negative for the antigen (0/11). The frequency of NY-ESO-1 in melanomas in the vertical growth phase was $24 \%$ $(16 / 68) .{ }^{71}$ Goydos et al. published, in 2001, a study in which 52 melanomas were analyzed, 20 of them were primary, 22 were locoregional metastases, and 10 were distant metastases for the presence of NY-ESO-1 by the PCR method. ${ }^{72}$ Overall, 33\% (17/52) of melanomas 
were antigen positive, most of them in locoregional and distant metastases. In 2006, Barrow et al. published a study of 586 melanoma samples from 426 individuals without previous selection and the immunohistochemical test was positive in $46 \%$ of cases, regardless of the disease stage. ${ }^{73}$ In 2007 , Velasquez et al. found $13 \%$ positivity in a study with 61 primary melanomas and 32\% in 63 metastatic melanomas, using the immunohistochemical test. ${ }^{74}$ An Australian study with 321 patients identified the NY-ESO-1 protein in $37 \%$ of cutaneous melanomas in stages I and II using the immunohistochemistry technique for their detection. ${ }^{75}$ Differences in the frequency of NY-ESO-1 among the findings of the different studies may be due not only to the stage of melanoma (primary $x$ metastatic) but also to the method used (PCR $x$ immunohistochemistry) and to the cut-off used to consider the tumor as positive or negative. Table 3 summarizes the findings regarding the expression of NY-ESO-1 in melanoma until the present.

This expression seems to be more frequent in tumors of more advanced stages, associated to the progression of melanocytic tumors. ${ }^{71}$ It is not possible, however, to determine if antigen expression causes melanoma to progress or if the tumor, when progressing, starts to presenting new antigens, including NY-ESO-1.

As for the distribution of the antigen in melanoma tumor cells, there is no concordance between the studies in the literature. Barrow et al., in 2006, ${ }^{73}$ found that the complete pattern was the rarest and the regional pattern was the most common in primary tumors. Velasquez et al., in $2007,{ }^{74}$ found $50 \%$ of scattered distribution, $13 \%$ of regional and $37 \%$ of complete distribution among NY-ESO-1 positive. Giavina-Bianchi et al., in 2015, observed $47 \%$ of regional distribution, $35 \%$ of complete and $18 \%$ of scattered distribution. This demonstrates that the expression of the NY-ESO-1 antigen is quite heterogeneous among patients, in the same patient at different stages (primary $\mathrm{x}$ metastasis) and even within the same tumor sample. It is not clear why this occurs, but a plausible explanation would be the fact that antigen expression can only occur from a particular stage of the tumor or can be disrupted at any given time, which could explain such disparate patterns of distribution. The percentage of melanoma cells expressing NY-ESO-1 also varied greatly in the sample studied: an equal number of cases $(41 \%)$ expressing the protein in a small percentage ( $2 \%$ to $20 \%$ ) and a high percentage $(60 \%)$ of tumor cells, and only $18 \%$ of the tumors presenting an intermediate percentage between $21-60 \%$. This fact further corroborates the observation above the heterogenicity of NYESO-1 expression in primary skin melanomas.

Although women do not display any normal tissue expressing NY-ESO-1 antigen, unlike men expressing it in the testis, there is no difference in protein expression in tumors between the sexes. ${ }^{57}$ There was no significant association between NY-ESO-1 and age at diagnosis of primary melanoma or skin phototype either at the site of tumor onset, according to two studies that attempted to correlate these variables. ${ }^{71,75}$

In the analysis of the histological type, the superficial extensive type was negatively associated with the presence of NY-ESO-1 when compared with other histological types together $(\mathrm{p}<0.02) \cdot{ }^{71}$ Another study, published in 2011, found no association between these two variables. ${ }^{75}$ Emerging knowledge of genomic differences between melanoma subtypes, with the extensive superficial type most often harboring the BRAFV600E mutation compared with the other melanoma subtypes, is interesting in this context.

There is an association between Breslow index and NY-ESO-1 expression in primary invasive skin melanomas $(\mathrm{p}=$ 0.007). ${ }^{71}$ Other similar results have been published. A study evaluated 251 primary melanomas and showed increased expression of NY-ESO-1 in the intermediate group between $1.1-4.0 \mathrm{~mm}$ thickness compared with the thinner ones, less than $1.1 \mathrm{~mm} \cdot{ }^{73}$ In another study, examining 61 primary skin melanomas, the mean thickness of the positive tumors was $4.7 \mathrm{~mm}$ versus $1.53 \mathrm{~mm}$ in the negative. ${ }^{74}$ More recently, in 2011, a survey of 321 melanomas showed a greater expression of NY-ESO-1 antigen with increased melanoma thickness. ${ }^{76}$

TABLE 3: Main findings regarding NY-ESO-1 in cutaneous melanoma

\begin{tabular}{|c|c|c|c|c|}
\hline Number/type of melanoma & Frequency of NY-ESO-1 & Diagnostic method & Associations found $(p<0.05)$ & Authors \\
\hline $\begin{array}{l}52 / 20 \text { primary, } \\
22 \text { locoregional } \\
\text { metastases and } \\
10 \text { distant metastases }\end{array}$ & $33 \%$ & PCR & & Goydos et al., 2001 \\
\hline $\begin{array}{l}251 \text { primary and } \\
335 \text { metastatic }\end{array}$ & $46 \%$ in general & IHC & $>$ Breslow & Barrow et al., 2006 \\
\hline 348 primary & $37 \%$ & IHC & $\begin{array}{l}>\text { Breslow } \\
<\text { RFS }\end{array}$ & Svobodova et al., 2011 \\
\hline 61 primary & $13 \%$ & IHC & $>$ Breslow & Velasquez et al., 2007 \\
\hline 63 metastatic & $32 \%$ & & & \\
\hline 89 primary & $19 \%$ & IHC & $\begin{array}{l}>\text { Breslow } \\
<\text { sup. ext. } \\
<\text { CD3+ }\end{array}$ & $\begin{array}{l}\text { Giavina-Bianchi et al., } \\
2015\end{array}$ \\
\hline
\end{tabular}

$\mathrm{IHC}=$ Immunohistochemistry; RFS = relapse free survival; sup. ext. $=$ Superficial extensive histological type 
When analyzing the presence of ulceration in the tumor in relation to the positivity of the NY-ESO-1 protein, two studies evidenced a tendency to positive association, but there was no statistical significance. ${ }^{71,75}$ The number of mitoses in melanoma was not associated with the presence of NY-ESO-1 in the only study in which it was tested.

Although investigations indicate that the presence of NYESO-1 is associated with thicker tumors, and these are more frequently causing metastasis, there was no statistical difference in sentinel lymph node status or in the development of metastases of patients in relation to the presence of the antigen. ${ }^{71,75}$

As for disease-specific survival of patients, most studies have associated the presence of the expression of the Cancer-Testis antigens to a worse prognosis in a variety of cancers, such as neuroblastoma, ovarian cancer, breast cancer, multiple myeloma, and others. ${ }^{77-80}$ In melanomas, some studies have demonstrated the same association, ${ }^{72-74}$ but others did not show statistical difference between the groups. ${ }^{71,75}$

\section{Antigen NY-ESO-1 and the immune response}

The NY-ESO-1 antigen is the most immunogenic of the Cancer-Testis antigens known to date. Humoral and cellular responses with CD4 and CD8 lymphocytes are observed in approximately 5\% to $15 \%$ of patients with different types of tumors. ${ }^{81,82}$ However, when analyzing patients with NY-ESO-1 positive tumors, this frequency rises to $50 \%{ }^{83}$

The humoral response to NY-ESO-1 is diverse in relation to the class of immunoglobulin synthesized, being IgG1, IgG2, IgG3 and/or IgG4, which are frequently detected simultaneously in the patients serum, and the IgG1 isotype is the most frequent of them. ${ }^{57}$ Titers against NY-ESO-1 can be very high, being detected at a dilution of 1/1,000,000 in the ELISA, although most are between 1/6,400 and $1 / 100,000$. No in vivo function for these antibodies has yet been demonstrated, as we know that their targets are not accessible, since the expression of NY-ESO-1 in tumor cells is cytoplasmic. They could be involved in assisting the initiation of the immune response by the formation of immunocomplexes with antigens released by necrotic or apoptotic tumor cells. ${ }^{84,85}$ It has also been observed that by removing the tumor by surgery, there is often a drop in antibody titers. ${ }^{86}$

It is clear that the expression of the NY-ESO-1 protein is not independently associated with the prognosis and that the tumor immunological microenvironment is a determining factor in the progression and evolution of the disease. Figures 2, 3 and 4 outline the classification of tumor-infiltrating lymphocytes (TIL), which is becoming increasingly important in the study of cancers.

Recently, one study concluded that high-grade tumor-infiltrating lymphocytes (brisk) in primary melanoma is associated with a lower risk of death regardless of the tumor characteristics that are currently used by the American Joint Committee on Cancer (AJCC). ${ }^{87}$ In other studies, the presence of TIL in melanoma gave patients better prognosis. ${ }^{10}$ The type of tumor-infiltrating lymphocyte in melanomas in the vertical growth phase showed no association with the presence of NY-ESO-1. Non-brisk TIL tumors were the ones that most commonly presented NY-ESO-1 (14/47), followed by tumors with brisk (TIL 1/13) and, finally, absent TIL (1/8). ${ }^{71}$ Another study also reported a heterogeneous association between TIL type and 77 NYO-ESO-1 positive melanomas: $57 \%$ had absent TIL; $38 \%$, non- brisk TIL; and 5\%, brisk TIL. Both studies show that tumor expression of NY-ESO-1 does not necessarily guarantee an effective immune response, which, perhaps, contributes to the absence of correlation with patient survival.

The correlation between local immune response in the primary tumor and the presence of NY-ESO-1 was also analyzed. The results showed a negative association between the antigen and the presence of CD3+ cells in TIL ( $p=0.017)$. In NY-ESO-1 positive melanomas, not only there were fewer CD3+ cells in TIL, but they were isolated $(p=0.009)$ or in small groups of 2 to 5 cells $(p=0.0125)$, contrasting with negative NY-ESO-1 tumors, which presented arrangements with large groups of 6 or more CD3+ cells in the infiltrate $(p=0.0001) .{ }^{71}$ Contrary to expectations, the presence of the antigen did not result in a greater influx of $\mathrm{CD} 3+$ lymphocytes in the tumor.

Several articles show association of CD8+FoxP3- cells in TIL with a better prognosis. ${ }^{12,19,88} \mathrm{CD} 8-\mathrm{FoxP} 3+$ cells do not correlate with

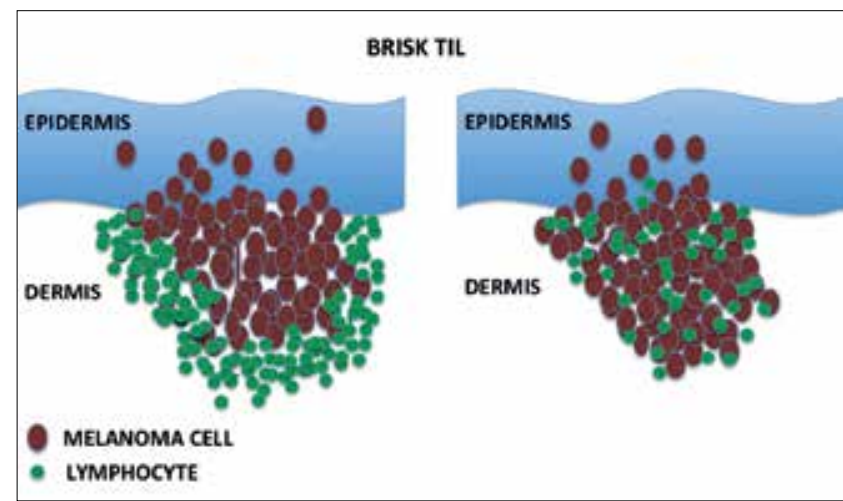

FIGURE 2: Schematic illustration of the brisk tumor-infiltrating lymphocytes

TIL is classified as "brisk" when the infiltrate around the vertical component of the tumor is continuous and consists of 4 or more rows of lymphocytes (left) or when it is located in large numbers, in close contact with the tumor cells (right)

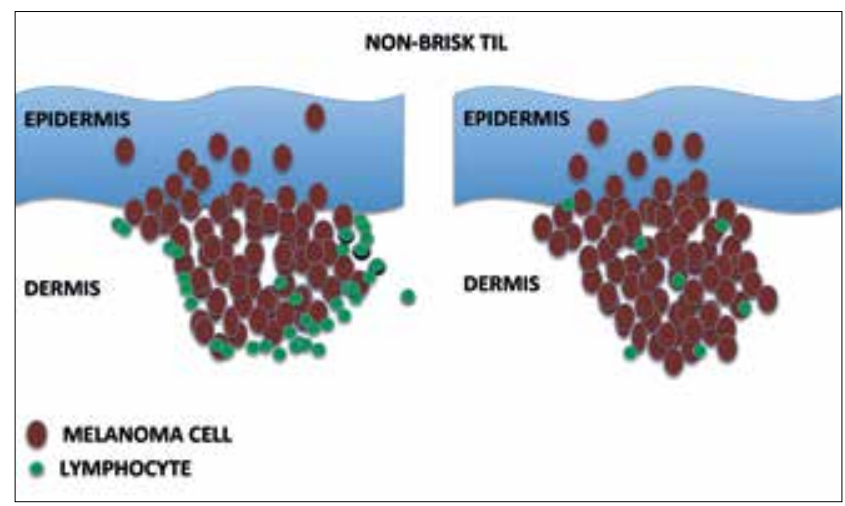

FIGURE 3: Schematic illustration of non-brisk tumor-infiltrating lymphocytes (TIL)

TIL is classified as "non-brisk" when the infiltration around the vertical component of the tumor is discontinuous or consists of less than 4 rows of lymphocytes (left) or when it is located in foci within the tumor, also in close contact with the tumor cells (right) 


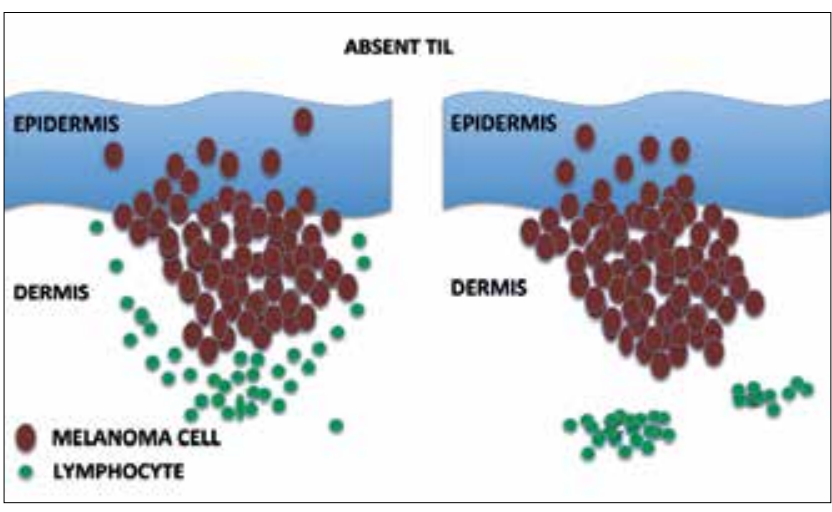

FIGURE 4: Schematic illustration of absent tumor-infiltrating lymphocytes (TIL)

TIL is classified as absent when the infiltrate around the vertical component of the tumor is not in close contact with tumor cells (left) or, when it does not exist, it is located around vessels or in areas of fibrosis (on the right)

survival in the literature, whereas CD8+/FoxP3+ ratio do, but they were analyzed only in a small number of cases. ${ }^{19,89}$ Giavina-Bianchi et al. found no association between the number of CD8+FoxP3-, CD8-FoxP3+ and CD8+FoxP3+ cells (double positive) in TIL and the expression of NY-ESO-1 in melanomas.

Immunological markers, such as the presence of circulating $\mathrm{T}$ cells specific for the NY-ESO-1 antigen, may be useful as a prognostic index of patients in the long term and also serve to monitor the response to immunotherapeutics. ${ }^{90}$ In 2008, Hunder et al. published a case report of refractory metastatic melanoma treated with autologous CD4+ cells stimulated in vitro with the NY-ESO-1 antigen, which resulted in complete tumor regression. ${ }^{91}$ In 2011, Robbins et al. observed regression of positive NY-ESO-1 metastatic melanomas treated with genetically modified lymphocytes to react to NY-ESO-1.92 In vitro studies have shown that there is tumor regression associated with specific immunity against NY-ESO-1, which correlated with a better prognosis for patients in clinical studies. ${ }^{58,60-62,64,93}$

NY-ESO-1 is considered the most promising antigen for the development of therapeutic vaccines. Many clinical trials using different formulations of NY-ESO-1 antigen (peptides, recombinant protein, bacterial and viral vectors associated with the use of different adjuvants) have been and are currently underway. ${ }^{94-99}$

\section{REFERENCES}

1. Linos E, Swetter SM, Cockburn MG, Colditz GA, Clarke CA. Increasing burden of melanoma in the United States. J Invest Dermatol. 2009;129:1666-74.

2. Armstrong BK, Kricker A. Cutaneous melanoma. Cancer Surv. 1994;19-20:219-40.

3. Inca.gov.br [Internet]. Câncer. Pele Melanoma. [acesso 15 jun 2016]. Disopnivel em: http://www.inca.gov.br/wps/wcm/connect/tiposdecancer/site/home/pele_ melanoma.

4. Seer.cancer.gov [Internet]. SEER Stat Facts Sheets: Melanoma of the Skin. [cited 2016 Jun 10]. Available from: http://www.seer.cancer.gov/statfacts/htlm/melan.htlm.

5. Baxevanis CN, Perez SA, Papamichail M. Combinatorial treatments including vaccines, chemotherapy and monoclonal antibodies for cancer therapy. Cancer Immunol Immunother. 2009;58:317-24.

6. Raval RR, Sharabi AB, Walker AJ, Drake CG, Sharma P. Tumor immunology and cancer immunotherapy: summary of the 2013 SITC primer. J Immunother Cancer. 2014;2:14

7. Romero P, Dunbar PR, Valmori D, Pittet M, Ogg GS, Rimoldi D, et al. Ex vivo staining of metastatic lymph nodes by class I major histocompatibility complex tetramers reveals high numbers of antigen-experienced tumor-specific cytolytic $T$ lymphocytes. J Exp Med. 1998;188:1641-50.
In these trials, one of the priorities has been the monitoring of patients' immune responses following immunizations. This immunological monitoring allows the comparison between the different assays and the identification of vaccine formulations and immunization strategies more effective and able to induce an integrated humoral and cellular response. ${ }^{100}$

A double-blind, randomized, placebo-controlled clinical trial used the recombinant NY-ESO-1 protein in conjunction with the ISCOMATRIX adjuvant (lipid/saponin-based encapsulating particles) for the immunization of patients with melanoma and induced immune response in all patients immunized with NY-ESO-1/ISCOMATRIX..$^{97-99}$ By using reinforcements with repeated injections of recombinant Fowlpox virus encoded with NY-ESO-1 in its entirety, the results obtained were even better. ${ }^{101}$ Other research has demonstrated effective inhibition of tumorigenesis and melanoma growth by using a novel NY-ESO-1 based vaccine and an aluminum-polysaccharide-HH2 adjuvant. ${ }^{102}$ Finally, a Brazilian study that promoted the blockade of CTLA4 and effected the vaccination with attenuated protozoa of Trypanosoma cruzi, expressing NY-ESO-1, was able to induce both the effector function of CD8+ lymphocytes and memory. ${ }^{103}$

\section{CONCLUSIONS}

We are living one of the most exciting periods in the study and development of drugs for metastatic melanoma. Seven new drugs have been approved by the FDA from 2011 to 2014 and others are in the process of being approved. The target therapies proved that metastatic melanoma regression was possible in many situations, but not lasting. On the other hand, inhibitors of the immune system checkpoints emerged as a new modality of treatment that, for a subgroup of patients, brought lasting clinical responses, an unprecedented fact. Toxicities secondary to drugs exist, but are manageable in most cases. Many combined or subsequent regimens of drugs are being conducted to see if there is increased benefit and/or decreased (or increased) adverse events.

A further range of options is opened with the discovery and production of vaccines from the Cancer-Testis antigens. The NYESO-1 antigen appears to be the most promising of them, with several studies and ongoing clinical trials. Finally, it is very important that we, dermatologists, know what is new in this area and that we also do not abstain from this fight against metastatic melanoma, in addition to the early diagnosis that already fits us.

8. Haanen JB, Baars A, Gomez R, Weder P, Smits M, de Gruijl TD, et al. Melanomaspecific tumor-infiltrating lymphocytes but not circulating melanoma-specific $T$ cells may predict survival in resected advanced-stage melanoma patients. Cancer Immunol Immunother. 2006;55:451-8.

9. Anichini A, Molla A, Vegetti C, Bersani I, Zappasodi R, Arienti F, et al. Tumorreactive $\mathrm{CD} 8+$ early effector $\mathrm{T}$ cells identified at tumor site in primary and metastatic melanoma. Cancer Res. 2010;70:8378-87.

10. Anichini A, Molla A, Vegetti C, Bersani I, Zappasodi R, Arienti F, et al. Tumor-infiltrating lymphocyte grade is an independent predictor of sentinel lymph node status and survival in patients with cutaneous melanoma. J Clin Oncol. 2012;30(21):2678-83.

11. Clemente CG, Mihm MC Jr, Bufalino R, Zurrida S, Collini P, Cascinelli N. Prognostic value of tumor infiltrating lymphocytes in the vertical growth phase of primary cutaneous melanoma. Cancer. 1996;77:1303-10.

12. van Houdt IS, Sluijter BJ, Moesbergen LM, Vos WM, de Gruijl TD, Molenkamp BG, et al. Favorable outcome in clinically stage II melanoma patients is associated with the presence of activated tumor infiltrating T-lymphocytes and preserved MHC class I antigen expression. Int J Cancer. 2008;123:609-15. 
13. Tuthill RJ, Unger JM, Liu PY, Flaherty LE, Sondak VK; Southwest Oncology Group. Risk assessment in localized primary cutaneous melanoma: a Southwest Oncology Group study evaluating nine factors and a test of the Clark logistic regression prediction model. Am J Clin Pathol. 2002;118:504-11.

14. Mihm MC Jr, Clemente CG, Cascinelli N. Tumor infiltrating lymphocytes in lymph node melanoma metastases: a histopathologic prognostic indicator and an expression of local immune response. Lab Invest. 1996;74:43-7.

15. Hillen $\mathrm{F}$, Baeten $\mathrm{Cl}$, van de Winkel $\mathrm{A}$, Creytens $\mathrm{D}$, van der Schaft DW, Winnepenninckx V, et al. Leukocyte infiltration and tumor cell plasticity are parameters of aggressiveness in primary cutaneous melanoma. Cancer Immunol Immunother. 2008:57:97-106.

16. Mandalà M, Imberti GL, Piazzalunga $\mathrm{D}$, Belfiglio $\mathrm{M}$, Labianca R, Barberis $\mathrm{M}$, et al. Clinical and histopathological risk factors to predict sentinel lymph node positivity, disease-free and overall survival in clinical stages I-II AJCC skin melanoma: outcome analysis from a single-institution prospectively collected database. Eur J Cancer. 2009;45:2537-45.

17. Bogunovic D, O'Neill DW, Belitskaya-Levy I, Vacic V, Yu YL, Adams S, et al. Immune profile and mitotic index of metastatic melanoma lesions enhance clinical staging in predicting patient survival. Proc Natl Acad Sci U S A. 2009;106:20429-34.

18. Burton AL, Roach BA, Mays MP, Chen AF, Ginter BA, Vierling AM, et al. Prognostic significance of tumor infiltrating lymphocytes in melanoma. Am Surg. 2011;77:188-92

19. Gooden MJ, de Bock GH, Leffers N, Daemen T, Nijman HW. The prognostic influence of tumour-infiltrating lymphocytes in cancer: a systematic review with meta-analysis. Br J Cancer. 2011;105:93-103

20. Cancer.gov [Internet]. Goverment U. Câncer treatment. [cited 2016 Jun 10]. Available from: http://www.cancer.gov/about-cancer/treatment/types/targetedtherapies/targeted-the.

21. Hocker TL, Singh MK, Tsao H. Melanoma genetics and therapeutic approaches in the 21st century: moving from the benchside to the bedside. J Invest Dermatol. 2008:128:2575-95

22. Chapman PB, Hauschild A, Robert C, Haanen JB, Ascierto P, Larkin J, et al. Improved survival with vemurafenib in melanoma with BRAF V600E mutation. N Engl J Med. 2011;364:2507-16.

23. Hauschild A, Grob JJ, Demidov LV, Jouary T, Gutzmer R, Millward M, et al. Dabrafenib in BRAF-mutated metastatic melanoma: a multicentre, open-label, phase 3 randomised controlled trial. Lancet. 2012;380:358-65.

24. Flaherty KT, Infante JR, Daud A, Gonzalez R, Kefford RF, Sosman J, et al. Combined BRAF and MEK inhibition in melanoma with BRAF V600 mutations. N Engl J Med. 2012;367:1694-703.

25. Flaherty KT, Infante JR, Daud A, Gonzalez R, Kefford RF, Sosman J, et al. Inhibition of mutated, activated BRAF in metastatic melanoma. N Engl J Med. 2012;367:1694-703

26. Schwartz RH. Costimulation of T lymphocytes: the role of CD28, CTLA-4, and B7/BB1 in interleukin-2 production and immunotherapy. Cell. 1992;71:1065-8.

27. Lenschow DJ, Walunas TL, Bluestone JA. CD28/B7 system of T cell costimulation. Annu Rev Immunol. 1996;14:233-58.

28. Rudd CE, Taylor A, Schneider H. CD28 and CTLA-4 coreceptor expression and signal transduction. Immunol Rev. 2009;229:12-26.

29. Freeman GJ, Gribben JG, Boussiotis VA, Ng JW, Restivo VA Jr, Lombard LA, et al. Cloning of B7-2: a CTLA-4 counter-receptor that costimulates human T cell proliferation. Science. 1993;262:909-11.

30. Azuma M, Ito D, Yagita $\mathrm{H}$, Okumura K, Phillips $\mathrm{JH}$, Lanier $\mathrm{LL}$, et al. $\mathrm{B} 70$ antigen is a second ligand for CTLA-4 and CD28. Nature. 1993;366:76-9.

31. Linsley PS, Brady W, Urnes M, Grosmaire LS, Damle NK, Ledbetter JA. CTLA-4 is a second receptor for the B cell activation antigen B7. J Exp Med. 1991:174:561-9.

32. Linsley PS, Clark EA, Ledbetter JA. T-cell antigen CD28 mediates adhesion with $B$ cells by interacting with activation antigen B7/BB-1. Proc Natl Acad Sci U S A. 1990;87:5031-5

33. Hodi FS, O'Day SJ, McDermott DF, Weber RW, Sosman JA, Haanen JB, et al. Improved survival with ipilimumab in patients with metastatic melanoma. N Engl $J$ Med. 2010:363:711-23.

34. Ribas A, Kefford R, Marshall MA, Punt CJ, Haanen JB, Marmol M, et al. Phase III randomized clinical trial comparing tremelimumab with standard-of-care chemotherapy in patients with advanced melanoma. J Clin Oncol. 2013:31:616-22.

35. Wolchok JD, Hoos A, O'Day S, Weber JS, Hamid O, Lebbé C, et al. Guidelines for the evaluation of immune therapy activity in solid tumors: immune-related response criteria. Clin Cancer Res. 2009;15:7412-20.

36. Pardoll DM. The blockade of immune checkpoints in cancer immunotherapy. Nat Rev Cancer. 2012;12:252-64.

37. Peggs KS, Quezada SA, Chambers CA, Korman AJ, Allison JP. Blockade of CTLA-4 on both effector and regulatory $\mathrm{T}$ cell compartments contributes to the antitumor activity of anti-CTLA-4 antibodies. J Exp Med. 2009;206:1717-25.
38. Ishida Y, Agata Y, Shibahara K, Honjo T. Induced expression of PD-1, a nove member of the immunoglobulin gene superfamily, upon programmed cell death. EMBO J. 1992;11:3887-95.

39. Keir ME, Butte MJ, Freeman GJ, Sharpe AH. PD-1 and its ligands in tolerance and immunity. Annu Rev Immunol. 2008;26:677-704.

40. Zou W, Chen L. Inhibitory B7-family molecules in the tumour microenvironment Nat Rev Immunol. 2008;8:467-77.

41. Topalian SL, Sznol M, McDermott DF, Kluger HM, Carvajal RD, Sharfman WH et al. Survival, durable tumor remission, and long-term safety in patients with advanced melanoma receiving nivolumab. J Clin Oncol. 2014;32:1020-30.

42. Dong H, Strome SE, Salomao DR, Tamura H, Hirano F, Flies DB, et al. Tumorassociated B7-H1 promotes T-cell apoptosis: a potential mechanism of immune evasion. Nat Med. 2002;8:793-800.

43. Konishi J, Yamazaki K, Azuma M, Kinoshita I, Dosaka-Akita H, Nishimura M. B7-H1 expression on non-small cell lung cancer cells and its relationship with tumor-infiltrating Iymphocytes and their PD-1 expression. Clin Cancer Res. 2004;10:5094-100.

44. Brown JA, Dorfman DM, Ma FR, Sullivan EL, Munoz O, Wood CR, et al. Blockade of programmed death-1 ligands on dendritic cells enhances $T$ cell activation and cytokine production. J Immunol. 2003;170:1257-66

45. Taube JM, Anders RA, Young GD, Xu H, Sharma R, McMiller TL, et al. Colocalization of inflammatory response with B7-h1 expression in human melanocytic lesions supports an adaptive resistance mechanism of immune escape. Sci Transl Med. 2012;4:127ra37.

46. Rosenwald A, Wright G, Leroy K, Yu X, Gaulard P, Gascoyne RD, et al. Molecular diagnosis of primary mediastinal $B$ cell lymphoma identifies a clinically favorable subgroup of diffuse large B cell lymphoma related to Hodgkin lymphoma. J Exp Med. 2003:198:851-62.

47. Herndon TM, Demko SG, Jiang X, He K, Gootenberg JE, Cohen MH, et al. U.S. Food and Drug Administration Approval: peginterferon-alfa-2b for the adjuvant treatment of patients with melanoma. Oncologist. 2012;17:1323-8.

48. Chen YT, Scanlan MJ, Sahin U, Türeci O, Gure A0, Tsang S, et al. A testicular antigen aberrantly expressed in human cancers detected by autologous antibody screening. Proc Natl Acad Sci U S A. 1997:94:1914-8.

49. Türeci O, Sahin U, Schobert I, Koslowski M, Scmitt H, Schild HJ, et al. The SSX-2 gene, which is involved in the $t(X ; 18)$ translocation of synovial sarcomas, codes for the human tumor antigen HOM-MEL-40. Cancer Res. 1996:56:4766-72.

50. Simpson AJ, Caballero OL, Jungbluth A, Chen YT, Old LJ. Cancer/testis antigens, gametogenesis and cancer. Nat Rev Cancer. 2005:5:615-25

51. Juretic A, Spagnoli GC, Schultz-Thater E, Sarcevic B. Cancer/testis tumourassociated antigens: immunohistochemical detection with monoclonal antibodies. Lancet Oncol. 2003;4:104-9.

52. Cta.Incc.br [Internet]. Cancer immunity. CT database [cited 2015 Jan 11]. Available from: $h$ ttp://www.cta.Incc.br/modelo.php?idgene $=101 \&$ idmeta $=11$

53. Marchand M, Weynants P, Rankin E, Arienti F, Belli F, Parmiani G, et al. Tumor regression responses in melanoma patients treated with a peptide encoded by gene MAGE-3. Int J Cancer. 1995;63:883-5

54. Thurner B, Haendle I, Röder C, Dieckmann D, Keikavoussi P, Jonuleit H, et al. Vaccination with mage-3A1 peptide-pulsed mature, monocyte-derived dendritic cells expands specific cytotoxic $T$ cells and induces regression of some metastases in advanced stage IV melanoma. J Exp Med. 1999;190:1669-78.

55. Bettinotti MP, Panelli MC, Ruppe E, Mocellin S, Phan GQ, White DE, et al. Clinical and immunological evaluation of patients with metastatic melanoma undergoing immunization with the HLA-Cw*0702-associated epitope MAGE-A12:170-178. Int J Cancer. 2003;105:210-6.

56. Jäger $E$, Chen $Y T$, Drijfhout JW, Karbach J, Ringhoffer $M$, Jäger $D$, et al. Simultaneous humoral and cellular immune response against cancer-testis antigen NY-ESO-1: definition of human histocompatibility leukocyte antigen (HLA)-A2-binding peptide epitopes. J Exp Med. 1998;187:265-70.

57. Gnjatic S, Nishikawa H, Jungbluth AA, Güre AO, Ritter G, Jäger E, et al. NY-ESO-1 review of an immunogenic tumor antigen. Adv Cancer Res. 2006:95:1-30.

58. Fujita $\mathrm{S}$, Wada $\mathrm{H}$, Jungbluth AA, Sato $\mathrm{S}$, Nakata T, Noguchi $\mathrm{Y}$, et al. NY-ESO-1 expression and immunogenicity in esophageal cancer. Clin Cancer Res. 2004;10:6551-8.

59. Peng JR, Chen HS, Mou DC, Cao J, Cong X, Qin LL, et al. Expression of cancer/ testis (CT) antigens in Chinese hepatocellular carcinoma and its correlation with clinical parameters. Cancer Lett. 2005;219:223-32.

60. Wang Y, Wu XJ, Zhao AL, Yuan YH, Chen YT, Jungbluth AA et al. Cancer/testis antigen expression and autologous humoral immunity to NY-ESO-1 in gastric cancer. Cancer Immun. 2004:4:11.

61. Nakada T, Noguchi Y, Satoh S, Ono T, Saika T, Kurashige T, et al. NY-ESO-1 mRNA expression and immunogenicity in advanced prostate cancer. Cancer Immun. 2003;3:10.

62. Odunsi K, Jungbluth AA, Stockert E, Qian F, Gnjatic S, Tammela J, et al. NYESO-1 and LAGE-1 cancer-testis antigens are potential targets for immunotherapy in epithelial ovarian cancer. Cancer Res. 2003;63:6076-83. 
63. Kurashige T, Noguchi Y, Saika T, Ono T, Nagata Y, Jungbluth A, et al. Ny-ESO-1 expression and immunogenicity associated with transitional cell carcinoma: correlation with tumor grade. Cancer Res. 2001;61:4671-4.

64. Jungbluth AA, Antonescu CR, Busam KJ, Iversen K, Kolb D, Coplan K, et al. Monophasic and biphasic synovial sarcomas abundantly express cancer/testis antigen NY-ESO-1 but not MAGE-A1 or CT7. Int J Cancer. 2001;94:252-6.

65. Hemminger JA, Ewart Toland A, Scharschmidt TJ, Mayerson JL, Kraybill WG, Guttridge DC, et al. The cancer-testis antigen NY-ESO-1 is highly expressed in myxoid and round cell subset of liposarcomas. Mod Pathol. 2013;26:282-8.

66. Mashino K, Sadanaga N, Tanaka F, Yamaguchi H, Nagashima H, Inoue H, et al. Expression of multiple cancer-testis antigen genes in gastrointestinal and breast carcinomas. Br J Cancer. 2001;85:713-20.

67. Kubuschok B, Xie X, Jesnowski R, Preuss KD, Romeike BF, Neumann F, et al. Expression of cancer testis antigens in pancreatic carcinoma cell lines, pancreatic adenocarcinoma and chronic pancreatitis. Int J Cancer. 2004;109:568-75.

68. Yin B, Zeng Y, Wang X, Liu G, Zhang M, Song Y. Expression and clinical significance of cancer-testis genes in clear cell renal cell carcinoma. Int J Clin Exp Pathol. 2014;7:4112-9.

69. Inaoka RJ, Jungbluth AA, Gnjatic S, Ritter E, Hanson NC, Frosina D, et al Cancer/testis antigens expression and autologous serological response in a set of Brazilian non-Hodgkin's lymphoma patients. Cancer Immunol Immunother. 2012;61:2207-14.

70. Lüftl M, Schuler G, Jungbluth AA. Melanoma or not? Cancer testis antigens may help. Br J Dermatol. 2004;151:1213-8.

71. Giavina-Bianchi M, Giavina-Bianchi P, Sotto MN, Muzikansky A, Kalil J, Festa-Neto $\mathrm{C}$, et al. Increased NY-ESO-1 expression and reduced infiltrating CD3+ T cells in cutaneous melanoma. J Immunol Res. 2015;2015:761378.

72. Goydos JS, Patel M, Shih W. NY-ESO-1 and CTp11 expression may correlate with stage of progression in melanoma. J Surg Res. 2001;98:76-80.

73. Barrow C, Browning J, MacGregor D, Davis ID, Sturrock S, Jungbluth AA, et al. Tumor antigen expression in melanoma varies according to antigen and stage. Clin Cancer Res. 2006;12:764-71.

74. Velazquez EF, Jungbluth AA, Yancovitz M, Gnjatic S, Adams S, O'Neill D, et al. Expression of the cancer/testis antigen NY-ESO-1 in primary and metastatic malignant melanoma (MM)--correlation with prognostic factors. Cancer Immun. 2007;7:11.

75. Svobodová S, Browning J, MacGregor D, Pollara G, Scolyer RA, Murali R, et al. Cancer-testis antigen expression in primary cutaneous melanoma has independent prognostic value comparable to that of Breslow thickness, ulceration and mitotic rate. Eur J Cancer. 2011;47:460-9

76. Vaughan HA, Svobodova S, Macgregor D, Sturrock S, Jungbluth AA, Browning $\mathrm{J}$, et al. Immunohistochemical and molecular analysis of human melanomas for expression of the human cancer-testis antigens NY-ESO-1 and LAGE-1. Clin Cancer Res. 2004;10:8396-404.

77. Oberthuer A, Hero B, Spitz R, Berthold F, Fischer M. The tumor-associated antigen PRAME is universally expressed in high-stage neuroblastoma and associated with poor outcome. Clin Cancer Res. 2004;10:4307-13.

78. Yakirevich E, Sabo E, Lavie 0, Mazareb S, Spagnoli GC, Resnick MB. Expression of the MAGE-A4 and NY-ESO-1 cancer-testis antigens in serous ovarian neoplasms. Clin Cancer Res. 2003;9:6453-60.

79. Krüger S, Ola V, Feller AC, Fischer D, Friedrich M. Expression of cancer-testis antigen CT7 (MAGE-C1) in breast cancer: an immunohistochemical study with emphasis on prognostic utility. Pathol Oncol Res. 2007;13:91-6.

80. Andrade VC, Vettore AL, Felix RS, Almeida MS, Carvalho F, Oliveira JS, et al. Prognostic impact of cancer/testis antigen expression in advanced stage multiple myeloma patients. Cancer Immun. 2008;8:2.

81. Stockert E, Jäger E, Chen YT, Scanlan MJ, Gout I, Karbach J, et al. A survey of the humoral immune response of cancer patients to a panel of human tumor antigens. J Exp Med. 1998;187:1349-54.

82. van Rhee F, Szmania SM, Zhan F, Gupta SK, Pomtree M, Lin P, et al. NY-ESO-1 is highly expressed in poor-prognosis multiple myeloma and induces spontaneous humoral and cellular immune responses. Blood. 2005;105(:3939-44.

83. Jäger $E$, Nagata $\mathrm{Y}$, Gnjatic $\mathrm{S}$, Wada $\mathrm{H}$, Stockert $\mathrm{E}$, Karbach J, et al. Monitoring CD8 T cell responses to NY-ESO-1: correlation of humoral and cellular immune responses. Proc Natl Acad Sci U S A. 2000;97:4760-5.

84. Albert ML, Sauter B, Bhardwaj N. Dendritic cells acquire antigen from apoptotic cells and induce class I-restricted CTLs. Nature. 1998;392:86-9.

85. Nagata Y, Ono S, Matsuo M, Gnjatic S, Valmori D, Ritter G, et al. Differential presentation of a soluble exogenous tumor antigen, NY-ESO-1, by distinct human dendritic cell populations. Proc Natl Acad Sci U S A. 2002;99:10629-34.

86. Jäger $\mathrm{E}$, Stockert $\mathrm{E}$, Zidianakis Z, Chen $\mathrm{YT}$, Karbach J, Jäger D, et al. Humoral immune responses of cancer patients against "Cancer-Testis" antigen NY-ESO-1: correlation with clinical events. Int J Cancer. 1999;84:506-10.
87. Thomas NE, Busam KJ, From L, Kricker A, Armstrong BK, Anton-Culver H, et al. Tumor-infiltrating lymphocyte grade in primary melanomas is independently associated with melanoma-specific survival in the population-based genes, environment and melanoma study. J Clin Oncol. 2013 Nov 20;31(33):4252-9.

88. Piras F, Colombari R, Minerba L, Murtas D, Floris C, Maxia C, et al. The predictive value of CD8, CD4, CD68, and human leukocyte antigen-D-related cells in the prognosis of cutaneous malignant melanoma with vertical growth phase. Cancer. 2005;104:1246-54.

89. Ma MW, Medicherla RC, Qian M, Vega-Saenz de Miera E, Friedman EB, Berman $\mathrm{RS}$, et al. Immune response in melanoma: an in-depth analysis of the primary tumor and corresponding sentinel lymph node. Mod Pathol. 2012;25:1000-10.

90. Weide B, Zelba H, Derhovanessian E, Pflugfelder A, Eigentler TK, Di Giacomo AM, et al. Functional T cells targeting NY-ESO-1 or Melan-A are predictive for survival of patients with distant melanoma metastasis. J Clin Oncol. 2012;30:1835-41.

91. Hunder NN, Wallen H, Cao J, Hendricks DW, Reilly JZ, Rodmyre R, et al. Treatment of metastatic melanoma with autologous CD4 + T cells against NY-ESO-1. N Engl J Med. 2008;358:2698-703.

92. Robbins PF, Morgan RA, Feldman SA, Yang JC, Sherry RM, Dudley ME, et al. Tumor regression in patients with metastatic synovial cell sarcoma and melanoma using genetically engineered lymphocytes reactive with NY-ESO-1. J Clin Oncol. 201;29:917-24.

93. Chitale DA, Jungbluth AA, Marshall DS, Leitao MM, Hedvat CV, Kolb D, et al. Expression of cancer-testis antigens in endometrial carcinomas using a tissue microarray. Mod Pathol. 2005;18:119-26.

94. Odunsi K, Matsuzaki J, Karbach J, Neumann A, Mhawech-Fauceglia P, Miller $A$, et al. Efficacy of vaccination with recombinant vaccinia and fowlpox vectors expressing NY-ESO-1 antigen in ovarian cancer and melanoma patients. Proc Natl Acad Sci U S A. 2012;109:5797-802.

95. Uenaka A, Wada H, Isobe M, Saika T, Tsuji K, Sato E, et al. T cell immunomonitoring and tumor responses in patients immunized with a complex of cholesterol-bearing hydrophobized pullulan (CHP) and NY-ESO-1 protein. Cancer Immun. 2007;7:9.

96. Kawabata R, Wada H, Isobe M, Saika T, Sato S, Uenaka A, et al. Antibody response against NY-ESO-1 in CHP-NY-ESO-1 vaccinated patients. Int J Cancer. 200;120:2178-84.

97. Maraskovsky E, Sjölander S, Drane DP, Schnurr M, Le TT, Mateo L, et al. NYESO-1 protein formulated in ISCOMATRIX adjuvant is a potent anticancer vaccine inducing both humoral and CD8+ t-cell-mediated immunity and protection against NY-ESO-1 + tumors. Clin Cancer Res. 2004;10:2879-90.

98. Chen $Q$, Jackson H, Parente P, Luke T, Rizkalla M, Tai TY, et al. Immunodominant CD4+ responses identified in a patient vaccinated with full-length NY-ESO-1 formulated with ISCOMATRIX adjuvant. Proc Natl Acad Sci U S A. 2004;101:9363-8.

99. Davis ID, Chen W, Jackson H, Parente P, Shackleton M, Hopkins W, et al. Recombinant NY-ESO-1 protein with ISCOMATRIX adjuvant induces broad integrated antibody and CD4(+) and CD8(+) T cell responses in humans. Proc Natl Acad Sci U S A. 2004;101:10697-702.

100. Old LJ. Cancer vaccines: an overview. Cancer Immun. 2008;8:1.

101. Chen JL, Dawoodji A, Tarlton A, Gnjatic S, Tajar A, Karydis I, et al. NY-ESO-1 specific antibody and cellular responses in melanoma patients primed with NYESO-1 protein in ISCOMATRIX and boosted with recombinant NY-ESO-1 fowlpox virus. Int J Cancer. 2015;136:E590-601.

102. Li M, Shi H, Mu Y, Luo Z, Zhang H, Wan Y, et al. Effective inhibition of melanoma tumorigenesis and growth via a new complex vaccine based on NY-ESO-1-alumpolysaccharide-HH2. Mol Cancer. 2014;13:179.

103. Dos Santos LI, Galvão-Filho B, de Faria PC, Junqueira C, Dutra MS, Teixeira $\mathrm{SM}$, et al. Blockade of CTLA-4 promotes the development of effector CD8+ $T$ lymphocytes and the therapeutic effect of vaccination with an attenuated protozoan expressing NY-ESO-1. Cancer Immunol Immunother. 2015;64:311-23.

\footnotetext{
MAILING ADDRESS:

Mara Huffenbaecher Giavina-Bianchi

Av. Dr. Enéas de Carvalho Aguiar, 255 - $3^{\circ}$ andar

Cerqueira César

05403-900 - São Paulo - SP

Brazil

Email:marahgbianchi@gmail.com
}

How to cite this article: Giavina-Bianchi MH, Giavina-Bianchi Junior PF, Festa Neto C. Melanoma: tumor microenvironment and new treatments. An Bras Dermatol. 2017;92(2):156-66. 


\section{QUESTIONS}

1. Regarding melanoma, it is CORRECT to state that:

A. mortality is increasing in white skin patients

B. incidence is declining

C. in Brazil, it is estimated that more than 50 thousand cases will be diagnosed in 2016

D. US data for 2016 estimate fewer new cases in that country than in Brazil

2. Mark the drug that is NOT on the list of drugs recently approved by the FDA (between 2011 and 2014):
A. Vemurafenib
B. Ipilimumab
C. Dacarbazine
D. Nivolumab

3. Regarding the immune system, it is CORRECT to state that:

A. CD4+ $\mathrm{T}$ cells bind to antigens presented by antigenpresenting cells through MHC class I molecules

B. CD8+ T cells bind to antigens presented by antigen-presenting cells through MHC class II molecules

C. NK (natural killer) cells are a type of regulatory T cell

D. CD4+ T cells can differentiate into helper T cells

4. In relation to tumor infiltrating lymphocyte and the prognosis of cancer patients, we can state that:

A. the presence of CD3+ cells in TIL does not confer longer patient survival

B. the presence of CD8+ cells in TIL confer longer patient survival

C. the presence of FoxP3+ cells in TIL confer shorter patient survival

D. the higher the CD8+/FoxP3+ ratio in TIL, the shorter the patient's survival

5. Mark the RIGHT alternative in relation to target therapies:
A. they act on nonspecific cancer molecules
B. they are designed or chosen to interact with a target
C. they are similar to standard chemotherapy
D. they are cytotoxic

6. Mark the CORRECT alternative for the most common genetic mutations associated with various types of melanomas:
A. acral - GNA11
B. uveal - NRAS
C. mucosal - c-KIT
D. cutaneous - c-KIT

7. Mark the CORRECT alternative in relation to Vemurafenib:

A. it is first-line therapy for metastatic melanoma with BRAFV600K mutation

B. it has no cutaneous adverse events

C. secondary drug resistance is a rare event

D. oral medication used twice daily

8. Mark the CORRECT alternative in relation to Dabrafenib:

A. it is first-line therapy line for metastatic melanoma with BRAF mutation

B. it is reversible and selective inhibitor for BRAFV600E mutation

C. it has no cutaneous adverse events

D. secondary drug resistance is a rare event
9. Mark the CORRECT alternative in relation to Trametinib:

A. it is used in the treatment for metastatic melanoma with BRAFV600K and V600E mutation

B. it was approved by ANVISA in 2012

C. secondary drug resistance is a rare event

D. oral medication used twice daily

10. Mark the CORRECT alternative in relation to Ipilimumab:

A. it blocks the T lymphocyte CTLA4 receptor and, in so doing, stimulates the patient's immune system to fight melanoma

B. it is an oral monoclonal antibody approved by ANVISA in 2012

C. a large proportion of treated individuals presented a prolonged response, with more than 2 years of survival

D. the adverse events are, in general, neutropenia cases

11. Mark the CORRECT alternative in relation to anti-PD-1 drugs:

A. only Pembrolizumab has been released so far by ANVISA

B. the PD-1 axis has a stimulatory role of autoimmunity in the healthy individual

C. adverse events are more severe than those of Ipilimumab

D. Nivolumab is one of the representatives of this group

12. Mark the CORRECT alternative in relation to anti-PD-L1 drugs:

A. melanoma tumor cells and ovarian cancer may have high PD-L2 expression

B. tumor cells from B-cell lymphomas can express PD-L1

C. these drugs do not prevent PD-1 from binding to PD-L2

D. 3 compounds have recently been approved by the FDA

13. Mark the CORRECT alternative in relation to Peginterferon alfa $2 b$ :

A. the change in the interferon molecule decreased its half-life

B. it is used intravenously once a week

C. patients studied had an increase in survival

D. the subgroups most benefited by the use were patients with ulcerated melanoma and/or with lymph nodes with microscopic involvement

14. Mark the CORRECT alternative in relation to the characteristics of the Cancer-Testis antigens:

A. they have no expression in normal individuals

B. they do not induce humoral immune response

C. they have aberrant expression in various types of cancer

D. they have not been studied in cancer vaccines

15. Mark the CORRECT alternative in relation to NY-ESO-1:

A. it is the only Cancer-Testis antigen discovered to date

B. it is uncommon in liposarcomas and synovial sarcoma

C. it has mainly cytoplasmic expression

D. it is not detected in stomach, lung and ovarian cancers

16. Mark the CORRECT alternative in relation to NY-ESO-1 antigen and melanocytic tumors:

A. they present in a small percentage of nevi

B. their expression is more common in metastatic melanomas than in primary ones

C. there is no expression in melanomas in situ

D. they are detected only by immunohistochemical examination 
17. Mark the CORRECT alternative in relation to NY-ESO-1 antigen and melanoma:

A. it is associated with the Breslow index

B. it presents homogeneous distribution in the tumor

C. it is associated with a greater number of CD3+ cells in TIL

D. it is associated with worse prognosis

18. Mark the CORRECT alternative in relation to NY-ESO-1 antigen and the melanoma immune response:

A. NY-ESO-1 antigen is the least immunogenic of the Cancer-Testis antigens

B. patients present humoral, but non-cellular response to NY-ESO-1

C. the function of the anti-NY-ESO-1 antibodies in vivo is already well established

D. when removing a focus of metastasis or tumor, the titers of the antibodies fall

19. In relation to tumor infiltrate lymphocyte (TIL), we can state that:

A. the non-brisk type consists of large numbers of lymphocytes interacting directly with the tumor cells within the tumor and or making a continuous and thick margin (4 or more rows) around the tumor, touching its cells

B. the brisk type only means foci of lymphocytes interacting with tumor cells

C. the presence of TIL gives a better prognosis to the patient

D. absent type means complete absence of lymphocytes in the tumor
20. Regarding the vaccines tested with NY-ESO-1, check the CORRECT alternative:

A. the association of repeated reinforcements with Fowlpox virus was not superior to the isolated use of the NY-ESO-1/ISCOMATRIX vaccine

B. NY-ESO-1 antigen seems to be the most promising for the development of a vaccine among the Cancer-Testis antigens

C. use of the adjuvant HH-2 (polysaccharide aluminum) in the NY-ESO-1 vaccine showed an increase in melanoma growth

D. the attenuated Trypanosoma cruzi protozoan expressing NY-ESO-1 was used in treatment against ovarian cancer

\section{Answer key}

Diabetes mellitus and the skin. An Bras Dermatol. 2017;92(1):8-20

\begin{tabular}{llll}
\hline & & & \\
$1 . \mathrm{B}$ & $6 . \mathrm{D}$ & $11 . \mathrm{D}$ & $16 . \mathrm{C}$ \\
2.D & $7 . \mathrm{B}$ & $12 . \mathrm{C}$ & $17 . \mathrm{B}$ \\
$3 . \mathrm{B}$ & $8 . \mathrm{D}$ & $13 . \mathrm{C}$ & $18 . \mathrm{C}$ \\
$4 . \mathrm{C}$ & $9 . \mathrm{A}$ & $14 . \mathrm{B}$ & $19 . \mathrm{C}$ \\
$5 . \mathrm{D}$ & $10 . \mathrm{D}$ & $15 . \mathrm{A}$ & $20 . \mathrm{D}$ \\
\hline
\end{tabular}

Papers

Information for all members: The EMC-D questionnaire

is now available at the homepage of the Brazilian Annals of

Dermatology: www.anaisdedermatologia.org.br. The dead-

line for completing the questionnaire is 30 days from the date

of online publication. 